\title{
Active LSP based structure for Holographic 3D Video Display
}

\author{
Hao WANG ${ }^{1, a}$, Ming-Hua ZHANG ${ }^{1, b}$, Chuan SHEN ${ }^{2, c}$ and Sui WEl ${ }^{1, d,{ }^{*}}$ \\ ${ }^{1}$ Key Laboratory of Intelligent Computing \& Signal Processing, Ministry of Education, Anhui \\ University, Hefei, 230039 China \\ ${ }^{2}$ Key Laboratory of Modern Imaging and Displaying Technology of Anhui Province, Anhui University, \\ Hefei, 230039 China \\ aP13201097@ahu.edu.cn, b975304806@qq.com, c shenchuan2502@163.com, dswei@ahu.edu.cn \\ *Corresponding author
}

Keywords: Holographic display, Liquid crystal on silicon, Localized surface plasmons, Phase modulation.

\begin{abstract}
A novel structure hybridized localized surface plasmons (LSP) with liquid crystals(LC) to offer active modulation on sub wavelength structure, it allow for CMOS-compatible fabrication is presented with theoretical explanations and the results of numerical simulation. Among the candidate devices for true holographic video display, liquid crystal on silicon (LCOS), combining large birefringence liquid crystal with CMOS-compatible technology, is an appropriate digital spatial light modulator (SLM) for the purpose. In this work, the top electrode of LCOS is replaced by LSP Fabry-Pe'rot (FP) structure, which interacts with incident light on sub-wavelength scales. While keeping the advantages of LCOS, its physical process is novel. Instead of accumulation during light passed, phase retardation is offered by an anomalous reflection with high-efficiency. Our investigations are numerically carried out by the commercial software CST Microwave Studios. Some thoughts and inchoate simulation results are shared here.
\end{abstract}

\section{Introduction}

Holography has been acknowledged as the most promising technology for 3D display as it can produce three-dimensional image of the scene with perfect realism. In order to reproducing the moving 3D scene dynamically, the move to video holography seems not only desirable but also inevitable. MIT Spatial Imaging Group headed by Stephen Benton brought holography from the age of film to the digital age in 1989 and this is a remarkably transition. Since then R\&D on the devices for true holographic video display is advanced $[1,2,3]$. Heart of the holographic video is the devices that modulate the light by some kinds of spatial light modulators (SLMs). Among these candidate devices, LCOS, which is marriage of liquid crystal due to their large birefringence and CMOS-compatible technology to provide electrically (or digitally without mechanical motion) addressed structure and the possibility for high spatial resolution, is one of appropriate digital SLM for the purpose.

Spatial resolution is an important performance characteristic of SLM to provide the desired viewing angle in holographic display. The pixel size of SLM needs to close to the wavelength of visible light scale to provide the desired viewing angle in holographic display [1,2]. However, Kent state university researchers have pointed that simply scaling the pixel size in the off-the-shelf LCOS down to one micrometer or being close to the wavelength of visible light scale would have very low diffraction due to the fringing field effects in relative think cell to meet the need of $2 \pi$ retardation [4].

Contract to the off-the-shelf LCOS, the different structure PSR-based (Propagating Surface Plasmons) LC SLMs were already demonstrated in lectures $[5,6]$. By using the PSR effect, an enhanced electro-optic response in thin intermediate medium layer can be attained and opens the possibility of reducing the fringing field effect. On the other hand the spatial resolution of the PSR-based SLMs is limited by the finite propagation length (several tens of micrometers) of the SPs and their development has been hampered by the fact that these devices are expected to have a 
relatively low resolution. In contrast to PSR, non-propagating surface plasmons, usually referred to as localized plasmons or sometimes localized surface plasmon resonance, can be excited in subwavelength. LSP in metallic nanostructures can offer large electromagnetic field enhancements, and nanometre-scale localisation electric fields.

In this work, the top electrode of LCOS is replaced by LSP FP structure, which interacts with incident light on sub-wavelength scales. Coupling of LSP could create new hybrid modes that cannot be supported by individual nanostructures, overcome some of the limitations of individual LSP, and open up possibilities for new applications and active control of plasmon resonances.

\section{Physics of LSP FP Structure}

It is well known that holography is based on the electromagnetic interference effect, therefore the flectional phase is most being concerned in our purpose. According to the plasmon hybridization model that the LSP response of nanostructures can be viewed as the collection of LSP arising from simpler geometries to form an interacting system [7], several mechanisms for the nontrivial phase shift at the reflecting interface are introduced in this section. This could be helpful for understanding the structure suggested by us.

\section{Phase Shift on a Metal Subwavelength Grating}

The phase change on a grating associated with the LSP effect is complicated due to the phase changes associated with the modulated grating surface. Theoretically, it has been noticed that when light impinges on the interface of two media, i.e., from denser to rarer medium there is a possibility of existence of surface waves (evanescent wave) in the rarer medium and the reflected beam undergoes a lateral displacement along the interface. This effect was first observed by Goos and H"'anchen [8] and the analogue of the Goos and H"anchen effect for SPP have been studied both theoretically and experimentally. The normal wave vector is imaginary when the metal with a negative permittivity constitutes one of the interface media. The imaginary wave vector means that the wave is evanescent and decays exponentially from the interface. According the Fresnel's coefficient of reflection between the dielectric medium 1 and 2 (the permittivity is $\varepsilon_{1}$ and $\varepsilon_{2}$, and the permeability is $\mu_{1}=\mu_{2}=1$ ) [9].

$$
r=\frac{1-\varepsilon_{1} k_{2}^{\perp} / \varepsilon_{2} k_{1}^{\perp}}{1+\varepsilon_{1} k_{2}^{\perp} / \varepsilon_{2} k_{1}^{\perp}}
$$

where, $k_{j=1,2}^{\perp}=\sqrt{k_{0}^{2} \varepsilon_{j}-k_{/ /}^{2}}$ represents the wave vector component along the normal of interface, here $k_{0}$ is the wave vector in free space and the $k_{/ /}$is the parallel wave vector component along the boundary. When the normal wave vector $k_{2}^{\perp}$ is imaginary, however, the reflection phase will deviate from 0 or $\pi$. Hence, the evanescent wave will play a crucial role in tailoring the reflection phase at the boundaries.

For light incidence upon a metal surface perforated with the periodic subwavelength apertures, the propagating and evanescent waves can be excited simultaneously and the reflection phase may be changed depending on interference of diffracted evanescent waves generated by subwavelength features at the surface.

\section{Phase Shift Due to Resonant Cavities}

The reflection phase at the boundary of a slab can also be induced, which is correlated with the oscillation of waves in the slab. Consider a dielectric slab of the permittivity $\varepsilon_{2}$ and thickness $\mathrm{h}$ sandwiched between two semi-infinite medium $1\left(\varepsilon_{1}\right)$ and $3\left(\varepsilon_{3}\right)$ (as shown in Fig.1). Here we use the TM-polarized light is incident with the incident angle $\theta$ upon the slab and transmits from the medium 1 to medium 3 . 


$$
r=\frac{r_{12}+r_{23} \exp \left(2 i k_{2}^{\perp} h\right)}{1+r_{12} r_{23} \exp \left(2 i k_{2}^{\perp} h\right)}
$$

where, $\mathrm{r}$ is the amplitude of the total reflected light, $r_{12}$ (or $r_{23}$ ) is determined according to the Fresnel's reflection coefficient between the medium $\varepsilon_{1}$ and $\varepsilon_{2}$ (or $\varepsilon_{2}$ and $\varepsilon_{3}$ )(see equation 1): $k_{2}^{\perp}$ is the normal wave vector component in the slab.

\section{Phase Shift in Hybrid LSP Fabry-Perot (FP) Cavities}

As mentioned in the introduction, the hybrid LSP FP cavities is usually composed of a thin metallic pattern (e.g., a periodic array of well-separated disks, patches, or stripes) and a back ground plane, separated by a dielectric spacer. There are several similar architectures under different name, such as metal-insulator-metal (MIM) waveguides, gap-surface plasmon[10], guided resonance reflective phase shifters[11], for selectively modifying the wavelength, amplitude, phase or/and its polarization of electromagnetic field. In such structures presenting an effective optical index depending on their geometrical parameters such as their depth, height, width and the optical index of the spacer, horizontal or vertical resonances in the dielectric spacer could be excited; the vertical spacer may behave as the FP-like cavities[12].

\section{Our Proposed Structure}

The schematic view of structure we proposed is shown in Fig.1. Here, an air-filled metallic rectangular strip grating and a planar metal film, with the metal thickness much larger than the skin depth are separated by intermediate medium layer (e.g., LC layer). The grating is characterized by the lattice period $d$, slit width $a$, slit length $h$, and spacer thickness $t$. The structure is illuminated by a TM-polarized light, where the permittivity of incident side is $\varepsilon_{d}=1$, the incident angle is $\phi$, and the magnetic field acts along the $z$ axis. In this work the relative permittivity of metals (i.e., gold) is described using the Drude dispersion. Here the dielectric constant of dielectric layers in the middle $\varepsilon_{m}=1-\omega_{p}^{2} / \omega(\omega+i \gamma)$, where $\omega_{p}=1.37 \times 10^{16} \mathrm{rad} / \mathrm{s}$ and $\gamma=5 \times 10^{13} \mathrm{HZ}$. The spacer consisting of active intermediate medium layer aims at the modulation of SPs supported at the interface of a metal and a nonlinear material by an external control signal. The top and bottom metal layers can be potentially integrated as electrodes in a straightforward manner in the electro-optic system.

For simplify, all the discussions are derived in the 1D case, a simplified formula will be derived as:

$$
\left\{\begin{array}{l}
H_{Z}^{I}(x, y)=e^{i k_{0}\left(\gamma_{0} x-u_{0} y\right)}+\sum_{m} R_{m} e^{i k_{0}\left(\gamma_{m} x+u_{m} y\right)}, \\
H_{Z}^{I I}(x, y)=A e^{-i q_{0} y}+B e^{i q_{0} y}, \\
H_{Z}^{I I I}(x, y)=\sum_{m}\left(T_{m} e^{-i k_{0} v_{m}(y+h)}+P_{m} e^{i k_{0} v_{m}(y+h)}\right) e^{i k_{0} \gamma_{m} x} .
\end{array}\right.
$$

where, $\gamma_{m}=\sqrt{\varepsilon_{1}} \sin \varphi_{i}+G_{m} / k_{0}, G_{m}=2 \pi m / d, q_{0}=k_{0} \sqrt{\varepsilon_{s}}, u_{m}=\sqrt{\varepsilon_{1}-\gamma_{m}^{2}}, v_{m}=\sqrt{\varepsilon_{3}-\gamma_{m}^{2}}, \varepsilon_{s}=\varepsilon_{s}^{0}(1+2 \delta / a)$. The rest of parameters are determined by the boundary conditions of the complete set of Maxwell equations or directly by Fresnel reflection coefficient. 


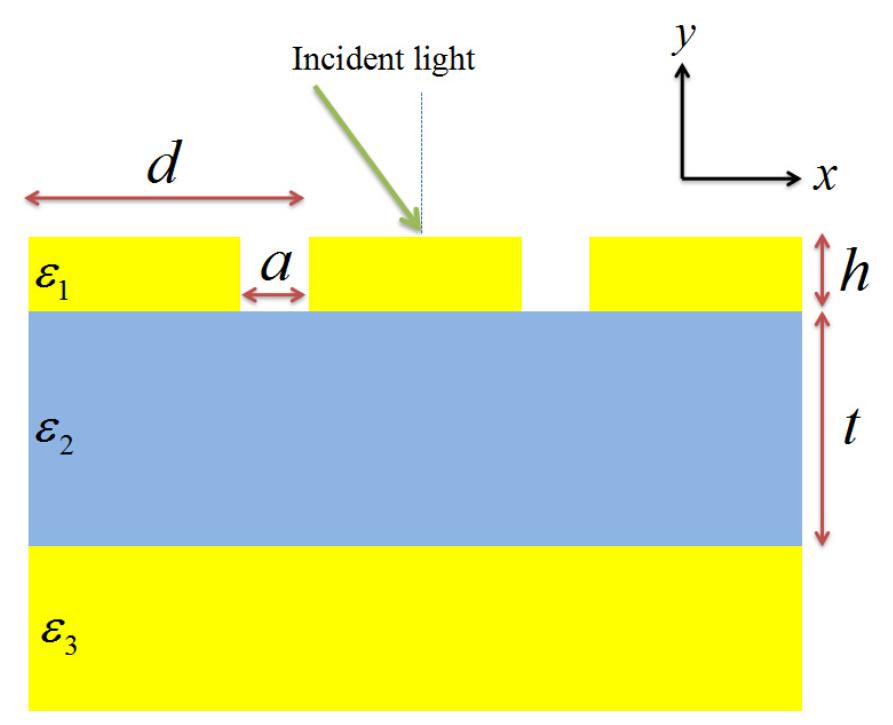

Fig.1 Schematic view of our proposed structure

\section{Numerical Simulations and Results}

To reveal the optical properties of our proposed structure, a simulation platform with the commercial software CST Microwave Studios and FDTD Solutions has been performed for our proposed structure in this paper. The structure is illuminated by a TM-polarized light. Here, without the loss of generality, our proposed structure parameters were set as lattice period $d=200 \mathrm{~nm}$, slit length $h=100$ $\mathrm{nm}$, slit width $a=50 \mathrm{~nm}$, the intermediate medium layer thickness $t=300 \mathrm{~nm}$. As shown in Fig.2(a), the phase of the reflected light is associated with the refractive index of the intermediate medium layer. We can see that with the increase of the reflective index of the intermediate medium layer, the reflected light phase displacement of monotone decreasing mode and the phase shift can reach to 357.302 degree.As shown in Fig.2(b), the phase shift can reach to 346.56 degree.
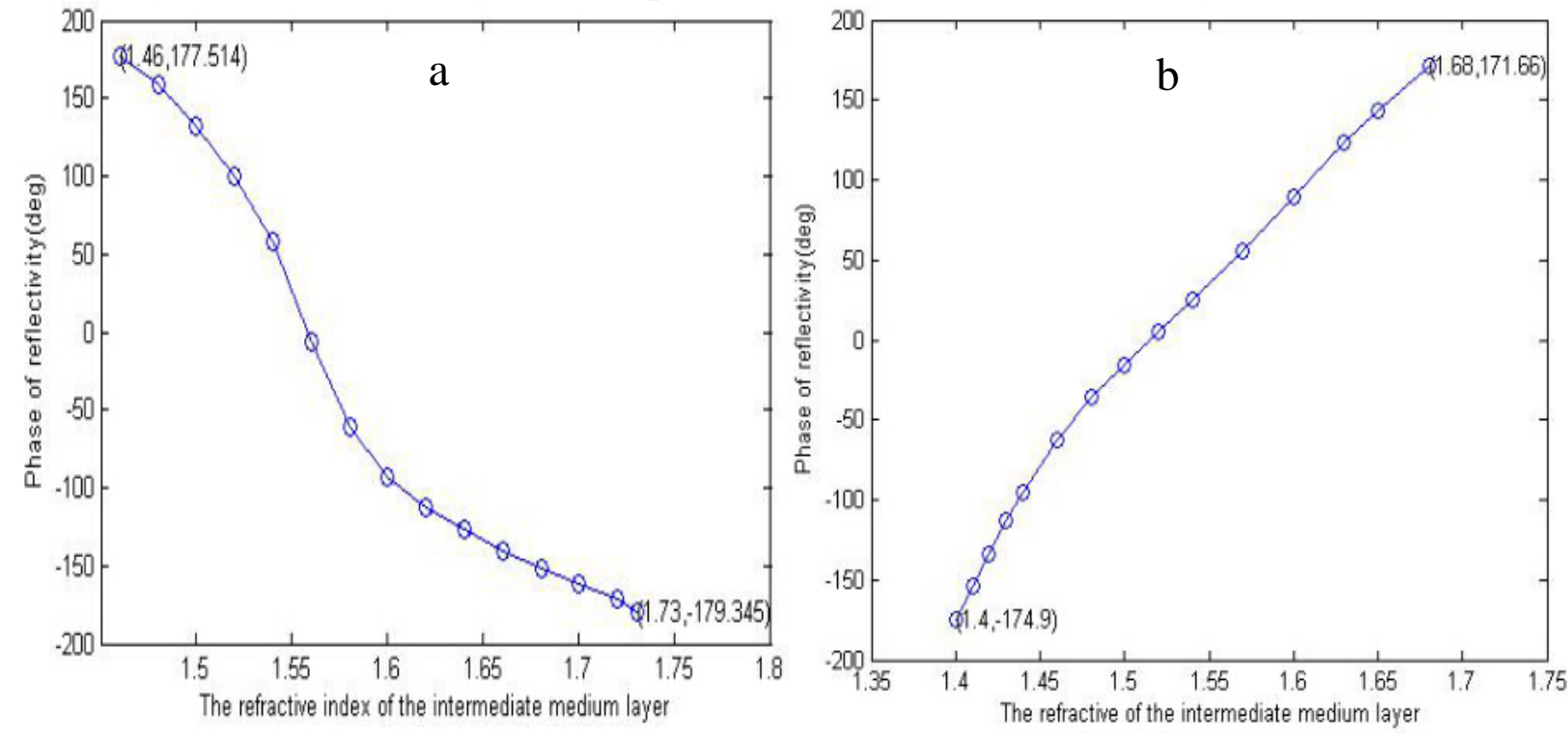

Fig. 2 Phase of the reflected light (a) CST Microwave Studios

(b) FDTD Solutions

Considering the industrial level, we improved our proposed structure. As shown in Fig. 3, if we want to carving slot depth is $100 \mathrm{~nm}$, there will be a certain slope the slope's width is about $10 \mathrm{~nm}$. As shown in Fig. 4(a), with the increase of the reflective index of the liquid crystal layer, the reflected light phase displacement of monotone decreasing mode and the phase shift can reach to 355.274 degree. As we know, a full $2 \pi$ phase shift is desired to generate an arbitrary wave front profile. For 
the above simulations, it is possible to approach the nearly $2 \pi$ phase region with our proposed structure. If the incident light energy unit is 1, as shown in Fig. 4(b), we can get that the amplitude of the reflected light lies between 0.78 and 0.92 .

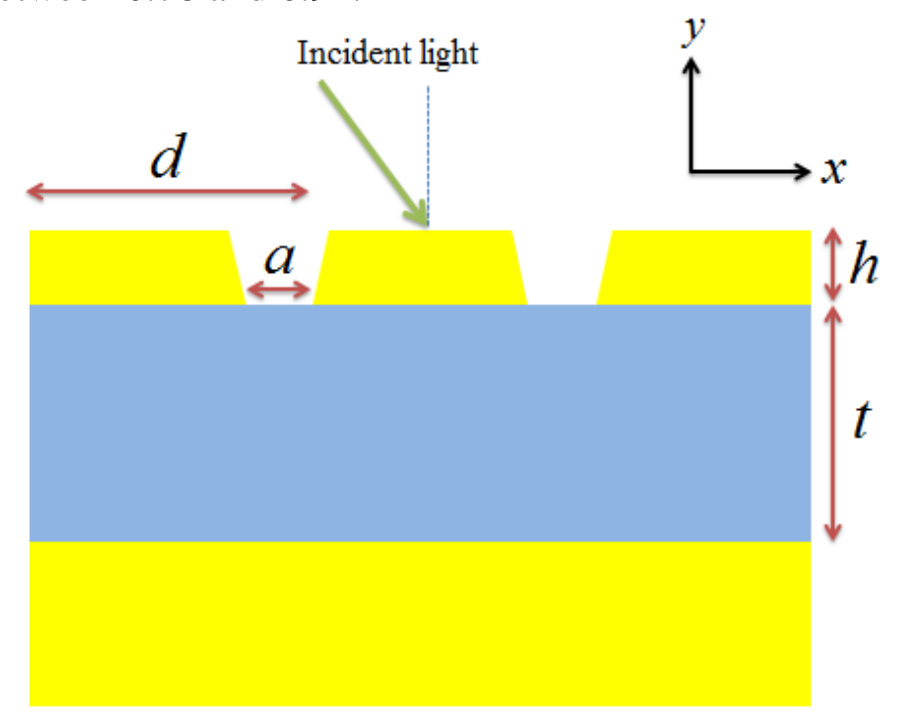

Fig. 3 Schematic view of our improved proposed structure

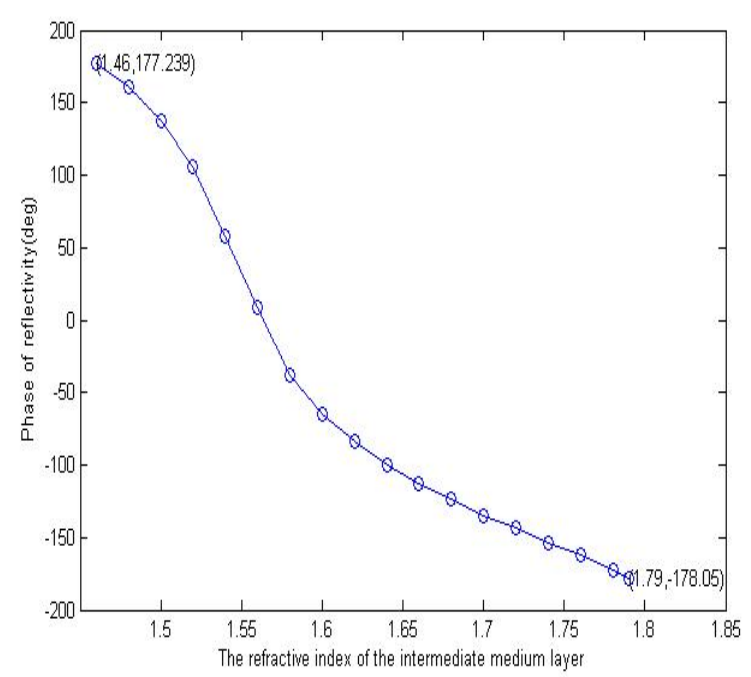

Fig. 4 (a) Phase of the reflected light.

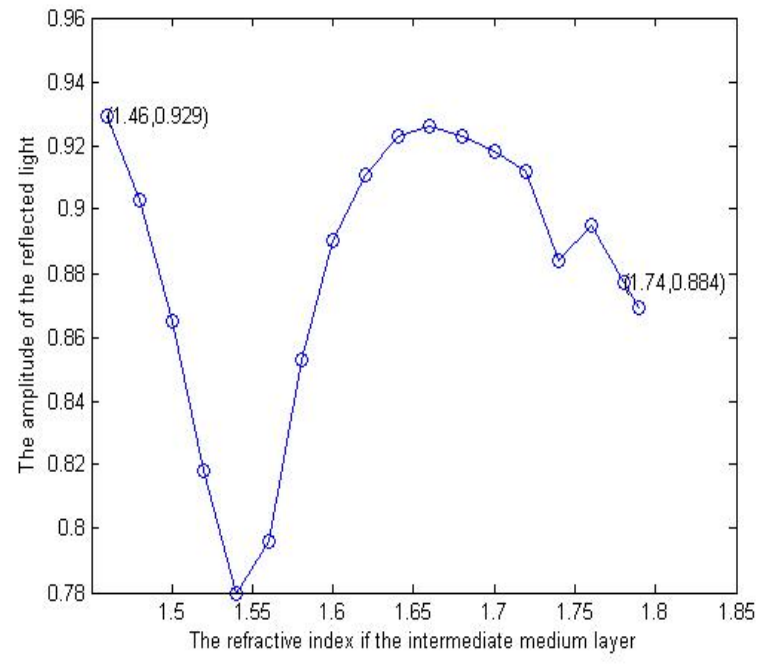

(b) The amplitude of the reflected light

\section{Conclusions}

A novel structure hybridized LSP with liquid crystal to offer active modulation on light-wavelength feature size and allow for CMOS-compatible fabrication is presented for holographic 3D video display. Its physical process is novel, instead accumulated during passing, phase retardation is offered by an anomalous reflection with phase shift in hybrid LSP FP cavities. As we all know, holography is the use of light interference principle record, diffraction theory reproducing the object information, including the recording information and reproducing the two processes. Holography recording process by interference principle record all the information (amplitude and phase information) of the object, so the holographic reproducing can be produced with the same original object wave-front, in order to achieve the object of the original three-dimensional reconstruction. Therefore, the phase modulation is what we are most concerned about.

In conclusion, The results obtained in the simulations are in good coincidence with the purposes of reaching nearly $2 \pi$ phase shift by change the dielectric constant of dielectric layers. At the same 
time it has a high reflection efficiency. Our results lead to more practical applications, in order to go beyond the limitations of current LCOS devices, improvements to existing LCOS devices. in future work we hope to achieve these experiments effects.

\section{Acknowledgement}

This work was supported by the National Natural Science Foundation of China (Grant Nos. 61377006, 61301296, 61201396, U1201255), Open Program of Key Laboratory of Opto-electronic Information Acquisition and Manipulation Ministry of Education (OEIAM201403, Anhui University). The authors would like to thank Cheng-Ping Huang from Department of Applied Physics, Nanjing Tech University for offering the commercial software CST Microwave Studios.

\section{References}

[1] Smalley D. E., Smithwick Q. Y. J., Bove V. M., Barabas, J. and Jolly, S., Anisotropic leaky-mode modulator for holographic video displays, Nature 498(7454), 313-317(2013).

[2] Stahl R., Rochus V., Rottenberg X., Cosemans, S., Haspeslagh, L., Severi, S. and Donnay, S., Modular sub-wavelength diffractive light modulator for high-definition holographic displays, J. Phys.: Conference Series 415, 012057(2013).

[3] Bleha W. P. and Lei L. J. A., Advances in Liquid Crystal on Silicon (LCOS) spatial light modulator technology, SPIE Defense, Security, and Sensing 23, 87360A(2013).

[4] Wang X., Wang B., Bos P. J., et al. Limitation of liquid crystal on silicon spatial light modulator for holographic three-dimensional displays, SID Symposium Digest of Technical Papers. Blackwell Publishing Ltd 35(1), 1522-1525(2012).

[5] Caldwell M. E. and Yeatman E. M., Surface-plasmon spatial light modulators based on liquid crystal, Applied Optics 31, 3880-3891(1992).

[6] Kogan P., Apter B., Baal-Zedaka I. and Efron U., Resolution improvement of surface plasmon-enhanced, liquid crystal spatial light modulator: Simulation studies, Optics Communications 281, 4788-4792 (2008).

[7] Prodan E., Radloff C., Halas N. J. and Nordlander P. A hybridization model for the plasmon response of complex nanostructures, Science 302: 419-422(2003).

[8] Goos F., and H“anchen H., Ein neuer und fundamentaler Versuch zur Totalreflexion, Ann. Phys. 436: 333-346(1947).

[9] Born M., and Wolf E., Principles of optics: electromagnetic theory of propagation, interference and diffraction of light, Cambridge U. Press, 1999.

[10] Pors A., Albrektsen O., Radko I. P., et al Gap plasmon-based metasurfaces for total control of reflected light, Scientific Reports, 3(7):2155-2155(2013).

[11] Yu H., Arbabi A., Faraon A., Guided resonance reflective phase shifters, SPIE OPTO. International Society for Optics and Photonics, 9372:93720W-1(2015).

[12] Cheng-Ping Huang, and Che-Ting Chan. Deep subwavelength Fabry-Perot resonances. Epj Applied Metamaterials 117(10):104102 (2015). 